\title{
TAVR in Patients on Hemodialysis: Outcome of A High-Risk Patient Group
}

\author{
Hendrik Ruge, ${ }^{1}$ Marcus-André Deutsch, ${ }^{2}$ Magdalena Erlebach, ${ }^{1}$ Melchior Burri, ${ }^{1}$ Sabine Bleiziffer, ${ }^{2}$ \\ Ruediger Lange ${ }^{1}$ \\ ${ }^{1}$ Department of Cardiovascular Surgery, German Heart Center Munich, TUM, Munich, Germany; ${ }^{2}$ Clinic for Thoracic and \\ Cardiovascular Surgery Heart and Diabetes Center NRW, Bad Oeynhausen, Germany
}

\section{ABSTRACT}

Background: Perioperative mortality is high and longterm survival is poor for patients on hemodialysis undergoing surgical aortic valve replacement (SAVR). Transcatheter aortic valve replacement (TAVR) offers a safe and effective therapy for high-risk patients suffering from aortic valve stenosis. However, in patients on hemodialysis only limited information is available on the outcome following TAVR.

Methods: Of the 2613 consecutive patients in our singlecenter TAVR registry, all hemodialysis patients, were identified. Demographics, procedural details, clinical outcomes, mortality, and complications were evaluated.

Results: Forty-two hemodialysis patients with a mean age of $75.2 \pm 8.2$ years, a mean STS predicted risk of mortality of $11.1 \pm 9.5 \%$ and a mean $\log$ EuroScore of $27.9 \pm 18.8 \%$ underwent TAVR. Mean duration on hemodialysis prior to intervention was $62.8 \pm 49.6$ months. A transfemoral access was chosen in 24 patients, a transapical in 16, and a transaxillary and a transaortic in one patient, respectively. Estimated survival at 30 days, one, three and five years was $83.3 \%, 68.3 \%, 37.7 \%$ and $18.9 \%$, respectively. Estimated median survival was $1.8 \pm 0.4$ years. VARC-2 defined perioperative complications included stroke in $7.1 \%$ (3/42), major bleeding in $16.7 \%(7 / 42)$, and vascular complications in $7.1 \%(3 / 42)$. In two patients, echocardiographic examination at three and four years, respectively, showed evidence for structural valve deterioration.

Conclusion: A high number of patients with ESRD undergoing TAVR require a non-transfemoral access. Predominantly, bleeding events contribute to the perioperative morbidity. An estimated median survival of less than two years after TAVR allows only limited assessment of valve prosthesis durability. Cardiovascular and non-cardiovascular mortality contribute equally to the causes of death beyond the first year after TAVR.

\section{INTRODUCTION}

Life expectancy is shortened significantly in patients with stage 5 renal disease, i.e. glomerular filtration rate

Received fune 4, 2020; received in revised form fune 29, 2020; accepted fune 29, 2020.

Hendrik Ruge, German Heart Center Munich, TUM, Munich, Germany (e-mail address: bendrikruge@aol.com). less than $15 \mathrm{ml} / \mathrm{min}$ per $1.73 \mathrm{~m}^{2}$ compared with individuals with normal kidney function [Gansevoort 2013]. The incidence of symptomatic aortic valve stenosis is $3.3 \%$ per year in patients on hemodialysis [Urena 1999]. Calcified aortic valve stenosis is the most common valvular heart disease in patients suffering from end-stage renal disease on hemodialysis [London 2000]. In these patients, high perioperative and late mortality and morbidity is reported after SAVR for aortic valve stenosis [Boning 2011; Thourani 2011; Thourani 2011; Williams 2016]. As TAVR has been shown to be equivalent or superior to SAVR in patients presenting with an intermediate or even low operative risk [Leon 2016; Mack 2019; Popma 2019; Reardon 2017], TAVR may offer a safe and effective therapy for hemodialysis patients with aortic valve stenosis. However, only limited information is available on survival, complications and bioprosthetic valve function after TAVR in patients with ESRD requiring hemodialysis [Allende 2014; Codner 2016; Dumonteil 2013; Ferro 2015; Ohno 2015; Szerlip 2016]. The objectives of this study were to evaluate (a) the early and late mortality and morbidity and (b) bioprosthetic valve function of patients with ESRD on hemodialysis after TAVR.

\section{MATERIAL AND METHODS}

Study design, patient population and follow up: Of 2,613 consecutive patients in our prospective single-center TAVR registry, all ESRD patients on hemodialysis were identified. Demographics, procedural details, clinical outcome, mortality and complications were prospectively recorded in a dedicated institutional database. Clinical follow-up visits were at 30 days, six and 12 months with yearly follow-up visits thereafter. Patients who were not seen at the local site were followed by telephone contact and information, such as ultrasound studies, were gathered from referring physicians. The institutional ethic committee approved the study.

Definitions: Postoperative outcomes were recorded according to the criteria of the updated Valve Academic Research Consortium (VARC-2) consensus document [Kappetein 2013]. Structural valve deterioration (SVD) and bioprosthetic valve failure were analyzed according to the EAPCI/ESC/EACTS definitions [Capodanno 2017]. If echocardiography studies did not report aortic valve gradients, qualitative assessments such as "normal function of the THV/no signs of elevated gradients" were accepted for bioprosthetic valve function assessment. 
Table 1. Baseline Characteristics

\begin{tabular}{lc}
\hline & N $=42$ patients \\
\hline Age, mean \pm SD & $75.2 \pm 8.2$ \\
Male (\%) & $29(69)$ \\
Severe pulmonary disease (\%) & $9(21.4)$ \\
Peripheral vascular disease (\%) & $24(57.1)$ \\
Porcelain aorta & $8(19.1)$ \\
Cerebrovascular disease (\%) & $3(7.1)$ \\
Severe pulmonary hypertension (\%) & $14(33.3)$ \\
Previous stroke (\%) & $2(4.8)$ \\
Coronary artery disease (\%) & $23(54.7)$ \\
Previous PCI (\%) & $11(26.2)$ \\
Previous CABG (\%) & $1(2.4)$ \\
Previous aortic valve surgery (\%) & $3(7.1)$ \\
Ejection fraction (\%) & \\
$>50 \%$ & $22(52.4)$ \\
$>35$ - 50\% & $7(16.7)$ \\
$<35 \%$ & $13(30.9)$ \\
STS PROM score, mean + SD & $11.1 \pm 9.5$ \\
Logistic EuroSCORE \% + SD & $27.9 \pm 18.8$ \\
NYHA functional class III or IV & $42(100)$ \\
Duration of dialysis (months) & $62.8+49.6$ \\
Mean \pm SD & \\
& \\
\hline &
\end{tabular}

Statistical analysis: Continuous variables are presented as means (standard deviation) or median (25th -75 th interquartile range), depending on the variable distribution. Categorical variables are presented as frequencies and percentages. Survival was assessed by the Kaplan-Meier estimator, with curves plotted along with the $95 \%$ confidence interval (CI). The SPSS statistical software package, Version 22 (IBM Corp., Armonk, NY, USA) was used for statistical analysis.

Results: Between July 2007 and March 2018, 42 hemodialysis patients (13 female, $31 \%$ ) underwent TAVR. Mean age was $75.2 \pm 8.2$ years, the mean STS predicted risk of mortality was $11.1 \pm 9.5 \%$ and the mean $\log$ EuroSCORE was 27.9 $\pm 18.8 \%$. Mean duration on hemodialysis prior to intervention was $62.8 \pm 49.6$ months. Comorbidities are summarized in Table 1 and included peripheral vascular disease in $57 \%$, coronary artery disease in $55 \%$, severe pulmonary hypertension in $33 \%$ (Table 1). In 24 patients a transfemoral, in 16 patients a transapical, in one patient a transaxillary and in one patient a transaortic access was chosen. From 2007 to 2014, in 20 ESRD-patients a transfemoral and transapical access was used in nine patients, and transaxillary and transaortic access in one patient. From 2015 to 2018 , in 15 patients a transfemoral and in seven patients a transapical access was used. Table 2 shows the spectrum of implanted transcatheter heart valves (THV) (Table 2).
Table 2. Procedural Details

\begin{tabular}{lc}
\hline Access route & N $=42$ patients \\
\hline Transfemoral (\%) & $24(57)$ \\
Transapical (\%) & $16(38.1)$ \\
Transaxillary (\%) & $1(2.4)$ \\
Transaortic (\%) & $1(2.4)$ \\
THV selection (\%) & \\
Medtronic Core Valve / Evolut R & $15(35.7)$ \\
Edwards Sapien / XT / 3 & $20(47.1)$ \\
Boston Scientific Lotus & $2(4.8)$ \\
Jena Valve & $2(4.8)$ \\
Symetis Accurate & $1(2.4)$ \\
St. Jude Medical Portico & $1(2.4)$ \\
Direct Flow & $1(2.4)$
\end{tabular}

Device success defined as absence of procedural mortality, correct positioning of one THV into proper anatomical location and normal bioprosthetic valve function was achieved in $92.9 \%$. In three patients, device success was not achieved due to a mean gradient $>20 \mathrm{mmHg}$ at discharge.

Survival: Perioperative and one-year mortality as well as mortality beyond one year after TAVR are summarized in Table 3. (Table 3) Estimated survival for 30 days, one, three and five years was $83.3 \%, 68.3 \%, 37.7 \%$ and $18.9 \%$, respectively. Estimated median survival was $1.8 \pm 0.4$ years (Figure 1 ). (Figure 1) Mean follow up was $568 \pm 618$ days.

Mortality and complications within 30 days post TAVR: Thirty-day mortality was $16.7 \%$ (7/42) with one non-cardiovascular death and six cardiovascular deaths. Septicemia lead to the non-cardiovascular death. Cardiovascular mortality included two strokes, ventricular arrhythmia/fibrillation, congestive heart failure, mesenterial ischemia, and one unknown cause of death.

VARC-2 defined perioperative complications included stroke in $7.1 \%(3 / 42)$, vascular complications in $7.1 \%(3 / 42)$, and major bleeding in $16.7 \%$ (7/42). Two patients had transfusion requirement, but no surgical intervention following transapical and transaortic access. Two patients underwent surgical intervention for bleeding after transfemoral access and following permanent pacemaker implantation. Three patients had transfusion requirement for gastrointestinal bleeding (two patients) or perioperatively for toxic megacolon surgery (one patient).

Follow up, complications and adverse events beyond one year after TAVR: Two patients were lost to follow up after TAVR at two months and two years, respectively. Among the patients $(\mathrm{N}=26)$ with at least one year follow up, seven died from cardiovascular cause, including five unknown causes of death and seven from non-cardiovascular cause. Three strokes were recorded 642, 1104, and 2273 days after TAVR, respectively. One patient suffered from a major bleeding event 379 days after TAVR. 


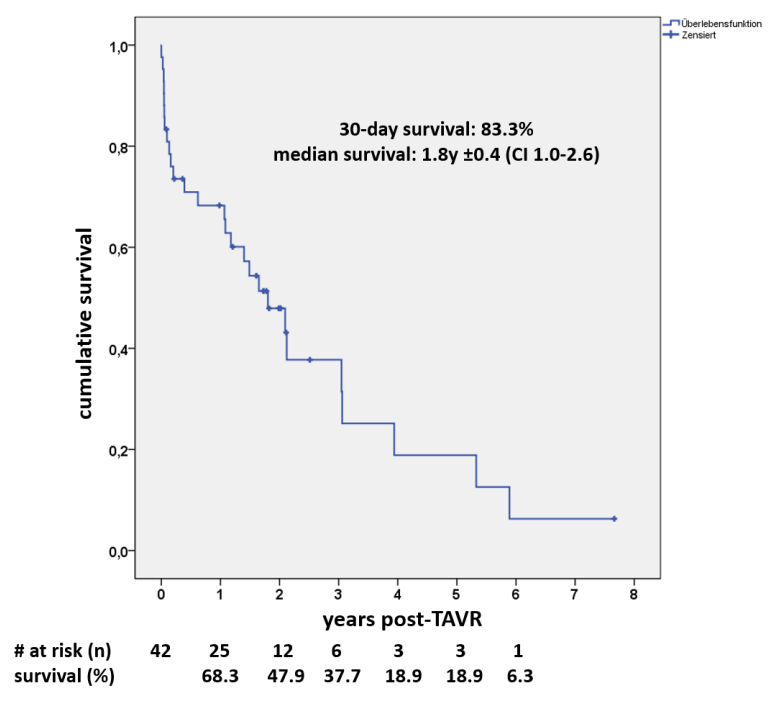

Figure 1. Estimated survival

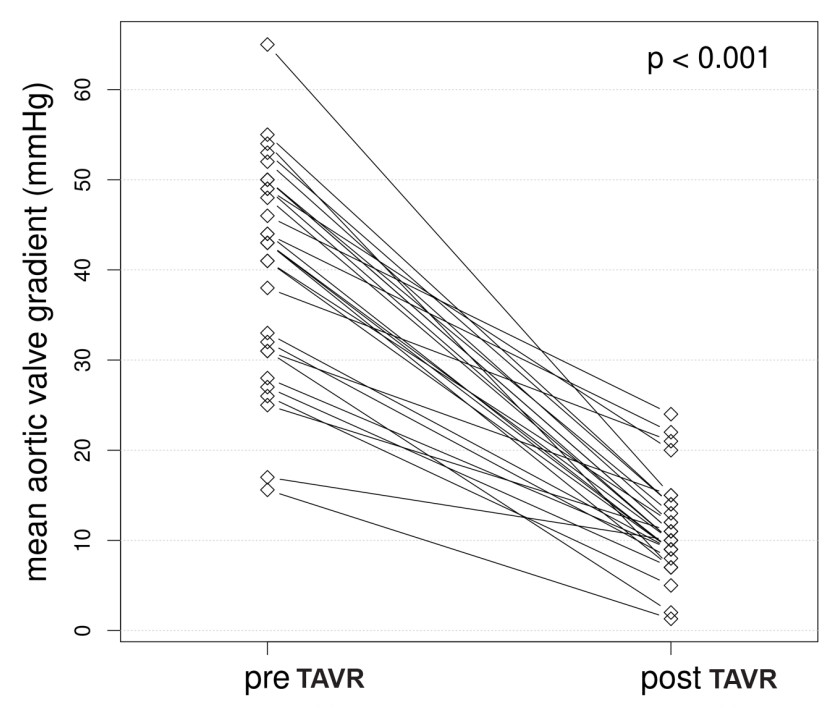

Figure 2. Mean aortic valve gradient

Hemodynamic performance and valve durability: At discharge, the mean transprosthetic pressure gradient (MPG) was $11.5 \pm 5.5 \mathrm{mmHg}$ (Figure 2) and remained stable throughout follow up (Figure 3). Three patients showed mild prosthetic aortic valve stenosis at discharge, according to VARC-2 definitions, with a mean gradient $>20 \mathrm{mmHg}$. In two of these patients, the mean transprosthetic gradient improved at sixmonth follow up and was below $20 \mathrm{mmHg}$, thus showing a normal prosthetic valve function, according to VARC-2 definitions. For the third patient, merely maximum transprosthetic gradients were available at follow up. These were

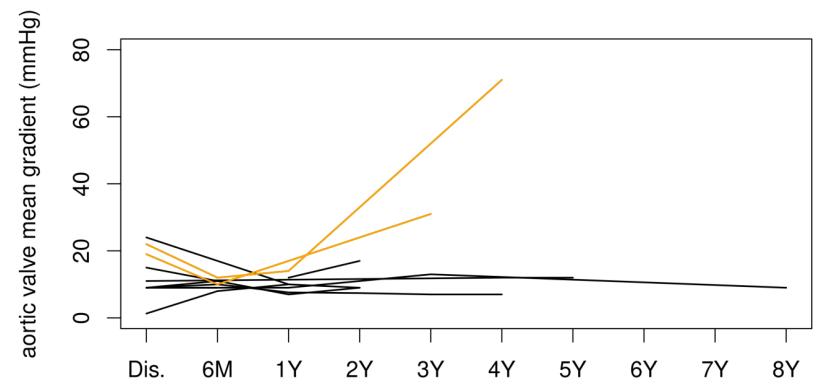

Figure 3. Aortic valve mean gradient

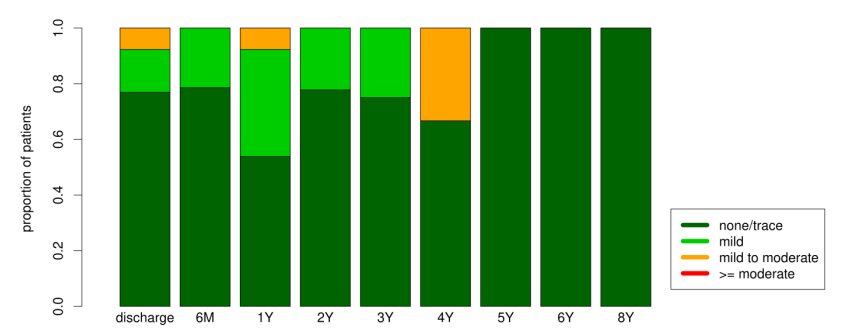

Figure 4. Paravalvular Leakage

similar to the discharge gradients (discharge: $38 \mathrm{mmHg}$, sixmonth follow up: $37 \mathrm{mmHg}$, one-year follow up: $43 \mathrm{mmHg}$ ).

Furthermore, during follow up, two patients presented with a mean gradient $>20 \mathrm{mmHg}$. One patient fulfilled the EAPCI/ECS/EACTS criteria [Capodanno 2017] for moderate hemodynamic structural valve deterioration (SVD) at three-year follow up, while the second patient fulfilled the criteria for severe hemodynamic SVD and thus met the criteria for bioprosthetic valve failure. Both patients died shortly after echocardiographic follow up had revealed SVD. The patient suffering from moderate SVD expired due to renal failure while the patient suffering from severe hemodynamic SVD died due to congestive heart failure.

In 39 patients, assessment of aortic regurgitation at discharge or 30-day follow up was available. Thirty patients showed none or trace paravalvular regurgitation (PVL). Mild PVL was found in three patients. Mild to moderate PVL was found in three patients (Figure 4). During follow up of the three patients with mild to moderate PVL, one patient died on day 18, another on 396 without further echocardiography follow up. The third patient showed mild PVL at six months, one- and two-year follow up, respectively.

Echocardiographic follow up beyond one year after TAVR revealed only one patient with mild to moderate PVL. The finding at one-year follow up improved to none/trace at three years, six years and eight years, respectively.

Bioprosthetic valve function assessment included quantitative echocardiographic measurements and qualitative echocardiographic descriptions, where quantitative measurements were missing. Qualitative assessment, such as "normal 
Table 3. Procedural Outcome

\begin{tabular}{|c|c|c|c|}
\hline Overall mortality (\%) & $7(16.7)$ & $13(30.9)$ & 14 \\
\hline Non-cardiovascular mortality (\%) & $2(4.8)$ & $6(14.3)$ & 7 \\
\hline Stoke (\%) & $3(7.1)$ & $5(11.9)$ & 3 \\
\hline Life-threatening (\%) & $1(2.4)$ & & \\
\hline Major (\%) & $6(14.3)$ & - & 1 \\
\hline Major vascular complication (\%) & $3(7.1)$ & & \\
\hline New pacemaker implantation (\%) & $3(7.1)$ & & \\
\hline
\end{tabular}

gradients," applied to one patient at 30-day follow up, one patient at six months follow up, one patient at one year and one patient at two-year follow up. No endocarditis occurred during follow up.

\section{DISCUSSION}

This study reports outcome beyond one year of ESRD patients undergoing TAVR. Thirty-day and one-year mortality is $16.7 \%$ and $30.9 \%$, respectively. Estimated median survival is $1.8 \pm 0.4$ years (CI 1.0-2.6) with a cumulative survival of $68.3 \%, 47.9 \%$ and $37.3 \%$ at one, two and three years, respectively. Life-threatening/major bleeding events were relatively high $(16.7 \%)$, while other VARC-complication rates compare to other TAVR cohorts. Not only were we able to achieve a high device success rate (92.9\%), but also only two patients showed signs of structural valve deterioration (4.8\%) during follow up.

ESRD patients undergoing SAVR show a high early mortality up to $22.7 \%$ [Boning 2011; Thourani 2011; Thourani 2011] and a poor long-term survival of $43 \%$ and $28 \%$ at three and five years, respectively [Thourani 2011]. Median survival ranges from 1.9 years-24.7 months (95 CI: 10.2-47.7 months) after SAVR in patients on hemodialysis [Boning 2011]. After TAVR, one-year mortality in patients on hemodialysis ranges from 24.2-54.8\% [Allende 2014; Codner 2016; Dumonteil 2013]. Our one-year mortality is slightly lower, but still in line with these results. After TAVR, thirty-day and one-year survival is significantly lower in ESRD patients compared with non-ESRD patients [Allende 2014; Codner 2016; Dumonteil 2013]. Early mortality of patients suffering from ESRD on hemodialysis is twofold if increased to early mortality of patients with normal renal function [Dumonteil 2013; Ferro 2015]. Accordingly, the early survival of patients on hemodialysis is poor compared with early mortality reported from national TAVR registries and recent randomized trials [Leon 2016; Mack 2019; Popma 2019; Reardon 2017; Holmes 2016; Walther 2015].

When comparing patients on hemodialysis undergoing TAVR or SAVR, the results are contradicting. Kobrin finds no significant differences in 30-day mortality (10\% TAVR vs. $8 \%$ SAVR) and one-year survival (60\% TAVR vs. $63.6 \%$ SAVR) in 194 propensity-matched patients [Kobrin 2015]. In contrast, Alqahtani finds significantly lower in-hospital mortality for patients on hemodialysis undergoing TAVR (6.1\%) than SAVR (13.7\%) in 197 propensity-matched patients [Alqahtani 2017].

Altogether, even with divergent results, it seems evident, that patients on hemodialysis benefit from TAVR, while having a low survival compared with the general TAVR population. Currently, available data on cause of death are limited and inconsistent. We predominantly find cardiovascular mortality contributing to early mortality, whereas cause of death beyond one year after TAVR is attributed equally to cardiovascular and non-cardiovascular mortality. Reasons for the higher rate of especially late mortality may be the reduced life expectancy of five years in ESRD patients older than 65 years [Gansevoort 2013]. Further research is needed to identify ESRD patients who will experience a survival benefit after treatment of a stenotic aortic valve.

While complications such as stroke or vascular complications are in line with data published from large TAVR cohorts and registries, bleeding complication rates were high in our trial. Life-threatening or major bleeding events (16.7\%) became evident predominantly in the early postoperative period. While this percentage seems high, other trials reporting on outcomes of patients on hemodialysis undergoing TAVR, report similar event rates.

Codner reports an almost eightfold increase in major bleeding events for patients on hemodialysis compared with patients with normal renal function [Codner 2016]. Accordingly, other multi-center studies report high life-threatening and major bleeding complication rates between $10.4 \%$ and $36.4 \%$ in patients on hemodialysis after TAVR [Allende 2014; Dumonteil 2013; Szerlip 2016; Conrotto 2017]. Interestingly, we saw that four out of seven bleeding complications had no relationship to the TAVR procedure but were attributed to secondary bleeding events. This suggests that patients on hemodialysis may in general be prone to more bleeding events. Up to date pathophysiological cascades, which may explain this, have not been fully understood. 
Preexisting peripheral vascular disease excluded a transfemoral access in 18 patients $(42.8 \%)$ of our cohort. Other cohorts also report high rates of non-transfemoral access (19.7-38.8\%) in TAVR patients on hemodialysis [Allende 2014; Codner 2016; Dumonteil 2013; Ferro 2015]. In comparison, unpublished data from our registry show a rate of $3.2 \%$ for non-transfemoral access in all TAVR patients 2017 $(\mathrm{N}=633)$, thus indicating that patients on hemodialysis more often require non-transfemoral access than the general TAVR population. As these patients required more often a nontransfemoral access, $3 / 42$ patients $(7 \%)$ experienced accessrelated bleeding complications (one transfemoral, one transapical, and one transaortic access). Third generation THV delivery systems presenting with reduced sheath dimensions, allow a transfemoral access even in small or moderately calcified access vessels [Ruge 2020], perhaps reducing complication rates and improving the outcome of ESRD patients.

We achieved device success, according to VARC-2 criteria in $92.9 \%$. This exceeds device success rates of $87.9 \%$ and $80.6 \%$ reported for other cohorts on hemodialysis [Allende 2014; Dumonteil 2013]. Noteworthy, Allende et al report device success rates around $80 \%$ for both, patients with normal renal function and patients on hemodialysis. In contrast, Dumonteil et al report > 94\% device success in patients with normal renal function or only slight renal impairment and a non-significant lower device success rate of $88 \%$ in patients on hemodialysis [Allende 2014; Dumonteil 2013]. Our high success rate may be due to the excellent hemodynamic results. This may be due to utilization of a supraannular valve in half of our patients.

Echocardiography demonstrated normal prosthetic valve function in all our patients at discharge with only three patients showing mildly elevated mean gradients, two of whom gained normal valve function during follow up. In our study, echocardiographic follow up revealed only three patients with PVL mild to moderate, all other patients had PVL $\leq$ mild. These PVL rates are lower than previously published data. TAVR in ESRD patients achieved low double digit mean transprosthetic gradients up to one year [Allende 2014; Codner 2016; Ohno 2015; Szerlip 2016], however 13\% to $19 \%$ > moderate PVL are reported at discharge and 30-day follow, respectively [Allende 2014; Dumonteil 2013; Szerlip 2016].

To our knowledge, there are only very limited data published on bioprosthetic valve function beyond one year after TAVR in patients on hemodialysis. We were only able to gather echocardiography data of approximately $50 \%$ of the surviving patients beyond one-year follow up. The low rate of echocardiographic follow up is due to several reasons, including comorbidities and high patient age. Similar challenges may contribute to $60 \%$ to $80 \%$ echocardiographic data completing the one-year follow up in other studies [Allende 2014; Szerlip 2016]. According to the new EAPCI/ESC/EACTS definition for valve dysfunction, we found structural valve deterioration in two patients at three- and four-year follow up, respectively (4.8\%) (mean follow-up 568 \pm 618 days). Aside from Conrotto, who reports $4.1 \%$ prosthetic valve dysfunction, according to VARC-2 criteria at $670 \pm 466$ days follow up in HD patients [Conrotto 2017], other studies did not report on prosthetic dysfunction.

In conclusion, patients on hemodialysis benefit from TAVR versus SAVR, while mortality remains high. They more commonly experience bleeding complications, perhaps due to the higher rate of non-transfemoral access due to preexisting comorbidities. Hemodynamic results are excellent, while long-term data is obviously missing as a result of the high mortality.

\section{STUDY LIMITATIONS}

The prospectively recorded data of our institutional dedicated TAVR database were retrospectively analyzed. Despite the use of the VARC criteria, some degree of observational bias must be expected as there was no independent event adjudification committee for this study. Complete echocardiographic data especially beyond one year after TAVR were difficult to obtain, limiting the conclusions for longterm hemodynamic results. Outcome of quality of life and heart failure symptoms could not be reported as only limited data could be gathered beyond 30-day follow up. Only two patients were lost to follow up, yet the cause of death remained unknown with some patients. However, the patient number presented represents one of the largest single center cohorts on TAVR patients on hemodialysis.

\section{CONCLUSION}

A significant number of patients with ESRD undergoing TAVR require a non-transfemoral access. Predominantly, bleeding events contribute to the perioperative morbidity. Patients on hemodialysis have a substantial operative risk and a considerable early and late mortality after TAVR and SAVR. Therefore, a lesser invasive TAVR procedure might be justified in these patients. An estimated median survival of less than two years of ESRD patients after TAVR allows only limited assessment of valve prosthesis durability. Further research is needed, to identify ESRD patients, who will experience a survival benefit after treatment of an aortic valve stenosis.

\section{REFERENCES}

Allende R, Webb JG, Munoz-Garcia AJ, et al. 2014. Advanced chronic kidney disease in patients undergoing transcatheter aortic valve implantation: insights on clinical outcomes and prognostic markers from a large cohort of patients. European Heart Journal 35:2685-96.

Alqahtani F, Aljohani S, Boobes K, et al. 2017. Outcomes of Transcatheter and Surgical Aortic Valve Replacement in Patients on Maintenance Dialysis. The American Journal of Medicine 130:1464.e1-1464.e11.

Boning A, Boedeker RH, Rosendahl UP, et al. 2011. Long-term results of mechanical and biological heart valves in dialysis and non-dialysis patients. The Thoracic and Cardiovascular Surgeon 59:454-9.

Capodanno D, Petronio AS, Prendergast B, et al. 2017. Standardized definitions of structural deterioration and valve failure in assessing longterm durability of transcatheter and surgical aortic bioprosthetic valves: 
a consensus statement from the European Association of Percutaneous Cardiovascular Interventions (EAPCI) endorsed by the European Society of Cardiology (ESC) and the European Association for Cardio-Thoracic Surgery (EACTS). European Journal of Cardio-Thoracic Surgery: Official Journal of the European Association for Cardio-Thoracic Surgery 52:408-417.

Codner P, Levi A, Gargiulo G, et al. 2016. Impact of Renal Dysfunction on Results of Transcatheter Aortic Valve Replacement Outcomes in a Large Multicenter Cohort. The American Journal of Cardiology 118:1888-1896.

Conrotto F, Salizzoni S, Andreis A, et al. 2017. Transcatheter Aortic Valve Implantation in Patients With Advanced Chronic Kidney Disease. The American Journal of Cardiology 119:1438-1442.

Dumonteil N, van der Boon RM, Tchetche D, et al. 2013. Impact of preoperative chronic kidney disease on short- and long-term outcomes after transcatheter aortic valve implantation: a Pooled-RotterdAm-MilanoToulouse In Collaboration Plus (PRAGMATIC-Plus) initiative substudy. American Heart Journal 165:752-60.

Ferro CJ, Chue CD, de Belder MA, et al. 2015. Impact of renal function on survival after transcatheter aortic valve implantation (TAVI): an analysis of the UK TAVI registry. Heart 101:546-52.

Gansevoort RT, Correa-Rotter R, Hemmelgarn BR, et al. 2013. Chronic kidney disease and cardiovascular risk: epidemiology, mechanisms, and prevention. Lancet 382:339-52.

Holmes DR, Jr., Nishimura RA, Grover FL, et al. 2016. Annual Outcomes With Transcatheter Valve Therapy: From the STS/ACC TVT Registry. The Annals of Thoracic Surgery 101:789-800.

Kappetein AP, Head SJ, Genereux P, et al. 2013. Updated standardized endpoint definitions for transcatheter aortic valve implantation: the Valve Academic Research Consortium-2 consensus document. The Journal of Thoracic and Cardiovascular Surgery 145:6-23.

Kobrin DM, McCarthy FH, Herrmann HC, et al. 2015. Transcatheter and Surgical Aortic Valve Replacement in Dialysis Patients: A Propensity-Matched Comparison. The Annals of Thoracic Surgery 100:1230-6; discussion 1236-7.

Leon MB, Smith CR, Mack MJ, et al. 2016. Transcatheter or Surgical Aortic-Valve Replacement in Intermediate-Risk Patients. The New England Journal of Medicine 374:1609-20.

London GM, Pannier B, Marchais SJ, Guerin AP. 2000. Calcification of the aortic valve in the dialyzed patient. Journal of the American Society of Nephrology: JASN 11:778-83.
Mack MJ, Leon MB, Thourani VH, et al. 2019. Transcatheter AorticValve Replacement with a Balloon-Expandable Valve in Low-Risk Patients. The New England Journal of Medicine.

Ohno Y, Attizzani GF, Barbanti M, et al. 2015. Transcatheter Aortic Valve Replacement for Severe Aortic Stenosis Patients Undergoing Chronic Dialysis. Journal of the American College of Cardiology 66:93-4.

Popma JJ, Deeb GM, Yakubov SJ, et al. 2019. Transcatheter Aortic-Valve Replacement with a Self-Expanding Valve in Low-Risk Patients. The New England Journal of Medicine.

Reardon MJ, Van Mieghem NM, Popma JJ, et al. 2017. Surgical or Transcatheter Aortic-Valve Replacement in Intermediate-Risk Patients. The New England Journal of Medicine 376:1321-1331.

Ruge H, Burri M, Erlebach M, Lange R. 2020. Access site related vascular complications with third generation transcatheter heart valve systems. Catheterization and Cardiovascular Interventions: Catheter Cardiovasc Interv. 2020;1-8. https://doi.org/10.1002/ccd. 29095.

Szerlip M, Kim RJ, Adeniyi T, et al. 2016. The outcomes of transcatheter aortic valve replacement in a cohort of patients with end-stage renal disease. Catheterization and Cardiovascular Interventions: Official Journal of the Society for Cardiac Angiography \& Interventions 87:1314-21.

Thourani VH, Keeling WB, Sarin EL, et al. 2011. Impact of preoperative renal dysfunction on long-term survival for patients undergoing aortic valve replacement. The Annals of Thoracic Surgery 91:1798-806; discussion 1806-7.

Thourani VH, Sarin EL, Keeling WB, et al. 2011. Long-term survival for patients with preoperative renal failure undergoing bioprosthetic or mechanical valve replacement. The Annals of Thoracic Surgery 91:1127-34.

Urena P, Malergue MC, Goldfarb B, Prieur P, Guedon-Rapoud C, Petrover M. 1999. Evolutive aortic stenosis in hemodialysis patients: analysis of risk factors. Nephrologie 20:217-25.

Walther T, Hamm CW, Schuler G, et al. 2015. Perioperative Results and Complications in 15,964 Transcatheter Aortic Valve Replacements: Prospective Data From the GARY Registry. Journal of the American College of Cardiology 65:2173-80.

Williams ML, Bavaria JE, Acker MA, et al. 2016. Valve Selection in EndStage Renal Disease: Should It Always Be Biological? The Annals of Thoracic Surgery 102:1531-1535. 